\title{
Three Criticisms of Newton's Inductive Argument in the Principia
}

\author{
Nicholas Maxwell \\ Science and Technology Studies, University College London, London, UK \\ Email: nicholas.maxwell@ucl.ac.uk
}

Received April 30 $0^{\text {th }}, 2013$; revised June $4^{\text {th }}, 2013$; accepted June $10^{\text {th }}, 2013$

\begin{abstract}
Copyright (C) 2014 Nicholas Maxwell. This is an open access article distributed under the Creative Commons Attribution License, which permits unrestricted use, distribution, and reproduction in any medium, provided the original work is properly cited. In accordance of the Creative Commons Attribution License all Copyrights (C) 2014 are reserved for SCIRP and the owner of the intellectual property Nicholas Maxwell. All Copyright (C) 2014 are guarded by law and by SCIRP as a guardian.
\end{abstract}

In this paper, I discuss how Newton's inductive argument of the Principia can be defended against criticisms leveled against it by Duhem, Popper and myself. I argue that Duhem's and Popper's criticisms can be countered, but mine cannot. It requires that we reconsider, not just Newton's inductive argument in the Principia, but also the nature of science more generally. The methods of science, whether conceived along inductivist or hypothetico-deductivist lines, make implicit metaphysical presuppositions which rigorously require us to make them explicit within science so that they can be critically assessed, alternatives being developed and assessed, in the hope that they can be improved. Despite claiming to derive his law of gravitation by induction from phenomena without resource to hypotheses, Newton does nevertheless acknowledge in the Principia that his rules of reasoning make metaphysical presuppositions. To this extent, Newton has a more enlightened view of scientific method than most 20th and 21st century scientists and historians and philosophers of science.

Keywords: Newton; Principia; Newton's Inductive Argument; Three Criticisms; Duhem; Popper; Metaphysical Presuppositions of Scientific Method; Theoretical Unity; New Conception of Science

\section{The Inductive Argument of the Principia}

In his Aim and Structure of Physical Theory, first published in 1906, Pierre Duhem ostensibly demolished Isaac Newton's inductive argument of his Principia, which claimed to have derived by induction the law of universal gravitation from the phenomena ${ }^{1}$. Duhem's criticism was echoed, and perhaps strengthened by Karl Popper. In this paper I make a suggestion as to how Duhem's and Popper's criticism may be countered. Implicit in work I have published over a number of decades there is, however, another criticism of Newton's argument which is more general, and applies to all conceptions of scientific method which specify empirical, and possibly simplicity, considerations that govern acceptance of laws and theories in physics, but which deny, or fail to acknowledge, that metaphysical hypotheses play a crucial role. This criticism is more difficult to counter.

In the Principia, Newton proceeds as follows. In Book 1, after defining crucial notions such as "quantity of motion", Newton formulates the following three laws of motion, the basis for classical mechanics ${ }^{2}$ :

1) Every body continues in its state of rest, or of uniform motion in a right line, unless it is compelled to change that state by forces impressed upon it.

2) The change of motion is proportional to the motive force impressed; and is made in the direction of the right line in

${ }^{1}$ Duhem ((1962: pp. 190-195), first published in French as a series of articles in 1904 and 1905, and as a book in 1906.

${ }^{2}$ Newton (1962: vol. 1, p. 13). which that force is impressed.

3) To every action there is always an opposed and equal reaction or the mutual actions of two bodies upon each other are always equal, and directed to contrary parts.

Newton then goes on in Book 1 to prove a great number of propositions and theorems, many, but by no means all, related to the task of establishing his universal law of gravitation and using it to explain the System of the World - that is, the solar system - to be taken up in Book 3. Thus the first theorem proves that a body attracted by a force to a fixed point moves in such a way that the line joining the body to the fixed point sweeps out equal areas in equal times - echoes of Kepler's 2nd law! Theorem 2 established the converse: if a body moves so that a line joining it to a fixed point sweeps out equal areas in equal times then it is attracted to the fixed point by a force. Proposition 11 establishes that a body moving in an ellipse experiences a force directed at a focus of the ellipse, the strength of the force being inversely proportional to the square of the distance. Newton goes on to establish similar results for bodies moving in hyperbolas and parabolas. He then goes on, in proposition 17 , to prove the converse of these results, namely that if a body moves under the influence of a force directed towards a fixed point, the force varies inversely as the square of the distance, then the body will move in a conic section-an ellipse, parabola or hyperbola.

Book 2 is in the main concerned with the motion of bodies through fluids. It may have been written in part to refute Descartes' vortex theory of the solar system, according to which 
invisible swirling matter in space sweeps the planets round the sun (a modified version of which was also held by Christiaan Huygens).

Book 3, exploiting the results of Book 1, sets out to establish Newton's universal law of gravitation and explain the System of the World. First, Newton makes explicit his conception of what we would today call "scientific method" in what he calls "Rules of Reasoning in Philosophy":

Rule 1: We are to admit no more causes of natural things than such as are both true and sufficient to explain their appearances.

Rule 2: Therefore to the same natural effects we must, as far as possible, assign the same causes.

Rule 3: The qualities of bodies, which admit neither intensification nor remission of degrees, and which are found to belong to all bodies within the reach of our experiments, are to be esteemed the universal qualities of all bodies whatsoever.

Rule 4: In experimental philosophy we are to look upon propositions inferred by general induction from phenomena as accurately or very nearly true, notwithstanding any contrary hypothesis that may be imagined, till such time as other phenomena occur, by which they may either be made more accurate, or liable to exceptions ${ }^{3}$.

Newton goes on to specify six "phenomena"-six regularities of the solar system - that form the empirical basis for arriving at the law of gravitation. These are that the moons of Jupiter and Saturn obey Kepler's 2nd and 3rd laws of planetary motion, and so too do the planets other than the earth in their motion round the sun; and our moon, in its motion round the earth, obeys Kepler's 2nd law.

Newton then goes on, in a number of propositions and theorems, to establish his universal law of gravitation by means of his laws of motion and mathematical theorems of Book I, and the six phenomena just indicated - the above four rules of reasoning being appealed to at various points. Thus Newton first proves, in Proposition 1, that the moons of Jupiter move subject to a force directed towards the centre of the planet that is inversely proportional to the square of the distance to the centre (i.e. $\mathrm{F} \propto 1 / \mathrm{D}^{2}$, where $\mathrm{D}$ is the distance from the centre of the moon to the centre of Jupiter). He goes on to establish the same for the moons of Saturn and, in Proposition 2, the same for the planets ( $\mathrm{D}$ in this case, of course, being the distance to the centre of the sun). The moon too is shown to obey the inverse square law (in Proposition 3). Then, invoking rules 1 and 2, Newton argues, in Proposition 4, that the force to which the moon is subject is the force of gravity - the very same force we feel on earth and call gravity, responsible for bodies falling near the earth's surface. Likewise (Proposition 5), the moons of Jupiter are drawn towards Jupiter by the force of gravitationas are the moons of Saturn towards Saturn. And indeed "there is a power of gravity tending towards all the planets". "And", Newton goes on "since all attraction (by Law III) is mutual, Jupiter will therefore gravitate towards all his satellites, Saturn towards his, and the earth towards the moon, and the sun towards the planets". And "all the planets do gravitate towards one another" which means, Newton points out, that Jupiter and Saturn, when closest together, will sensibly disturb each other's motion, as the sun disturbs our moon's motion, and the sun and moon disturb our sea (causing the tides). Then (in Proposition 6), Newton sets out to establish that "all bodies gravitate to-

${ }^{3}$ Newton (1962: Vol. 2, pp. 398-400). These rules were modified, and even added to, by Newton in successive editions of the Principia. wards every planet", weights of bodies, at any given distance from the centre of the planet, being proportional to the quantity matter (i.e. the mass). Newton then establishes that "there is a power of gravity pertaining to all bodies, that is proportional to several quantities of matter which they contain". Then, in Proposition 8, we have the theorem that two homogeneous spheres attracting each other by gravitation, the weight of either "will be inversely as the square of the distance between their centres". Newton then establishes that the centre of the solar system is, not the centre of the sun, but rather the centre of gravity of the solar system, the sun being somewhat in motion with respect to this centre as it is tugged this way and that by the gravitational attraction of the planets. Newton then derives Kepler's laws for the planets a priori as he puts it, the planets only moving precisely in ellipses, however, if gravitational forces between planets are neglected, and the sun is assumed not to move ${ }^{4}$.

Newton goes on to derive various consequences from his law of gravitation and what has been established so far. He discusses the flattening of the earth and other planets at the poles because of their rotation; variation in weight at different latitudes on earth; gravitational attraction of the moon and sun producing the tides; the motion of the moon, affected by gravitational attraction of both earth and sun-a difficult 3-body problem which cannot be solved exactly; the motion of comets, which are shown to be along conic sections (approximately parabolas close to the sun $)^{5}$.

It hardly needs to be said that what Newton does in the Principia is extraordinarily impressive. It really does seem that Newton derives his universal law of gravitation from the phenomena, just as he claimed he had done. First, there are the purely mathematical theorems: bodies that move so at to obey Kepler's laws must be deflected from uniform motion in a straight line by a force that varies inversely as the square of the distance. Then, observation tells us that moons and planets do actually move so as to obey Kepler's laws. Therefore they must be subject to a force that varies inversely as the square of the distance. And since we can move, by degrees, from the motion of stone thrown on earth to the motion of the moon round the earth, this force must be the force of gravitation, of which we are so familiar here on earth. Granted that every body in the universe gravitationally attracts every other body, it is clear that the motions of the moons and planets must deviate slightly from perfect Keplerian motion due to mutual gravitational attraction - the final, devastatingly convincing evidence in support of Newtonian theory.

\section{Duhem's Criticism of Newton}

But all this is, on the face of it, decisively demolished by Pierre Duhem. In 1906 in his The Aim and Structure of Physical Theory Duhem pointed out that Newton could not possibly have derived his law from the phenomena since, as Newton had himself pointed out, his law revealed that the phenomena are not correct. Because planets attract each other and the sun, Kepler's laws are not precisely obeyed. But what this means is that the whole argument of the Principia is nothing more than a reductio ad absurdum. Assume A (the laws of motion and Ke-

\footnotetext{
${ }^{4}$ All this is established in the first 24 pages of Book 3 of the Principia: see Newton (1962: Vol. 2, pp. 399-422).

${ }^{5}$ For a recent, impressive and very detailed account of Newton's inductive argument in the Principia see Harper (2011).
} 
pler's laws); derive B (Kepler's laws are false). B contradicts A, therefore A is false. Newton's great argument is reduced to the demonstration that his initial assumptions amount to a contradiction - not what Newton claimed to prove at all ${ }^{6}$.

Duhem's argument is pretty devastating. But one feels that it somehow does not quite do justice to the sophistication of Newton's argument. This argument is not, after all, a straightforwardly deductive argument. It is an argument that comes in a number of stages, some deductive, some inductive. And that the conclusion - the universal law of gravitation-leads to a correction in the premises-Kepler's laws - far from sabotaging the whole validity of the argument, is actually one of the great strengths of the argument, and provides additional strong grounds for holding that the law of gravitation should be accepted. It is almost, one might say, the clinching argument.

How can one salvage Newton's great argument from Duhem's attack? It can be done, perhaps, along the following lines. Newton argues that planets that move around a fixed sun in orbits that obey Kepler's laws are subject to a force that varies inversely as the square of the distance. We may take that part of the argument as deductive and valid. We then contribute the third law of motion (every force has its equal and opposite force) and, eventually, the law that every body attracts every other as the inverse square of the distance - arrived at by induction. This means that the planets do not move around the sun in accordance, precisely, with Kepler's laws (because they attract the sun and each other). Does this mean the deductive part of this argument is invalid? No, it does not. The argument is valid, but the premises of the argument are false. If the planets move in Keplerian orbits round a fixed sun, then indeed, quite validly, they are subject to the relevant force directed at the sun. It is just that the sun is not fixed, the planets attract it, and attract each other. So, the premise of the argument is false, but this deductive part of the argument is valid.

We can now go on to consider: Does the falsity of the premise - the strict falsity of Kepler's laws - invalidate Newton's entire argument? Well, hardly. Because if we feed in the more precise, the true (or very nearly true) versions of the premisesorbits very nearly Keplerian, but with deviations or "perturbations", due to mutual gravitational attraction of heavenly bodies, and run the entire argument as before, deductive stages plus inductive stages, with these new corrected premises, no Duhem inconsistency between premises and conclusion emerges at all. The whole argument becomes self-consistent.

It is the possibility of correcting the entire argument in this fashion, as a result of what has been learned from the first runthrough of the argument, that salvages Newton's whole argument, deductive and inductive, from Duhem's apparently devastating attack.

If Newton's argument was wholly and entirely deductive in character, Duhem's criticism would be more serious. In fact the argument has a number of stages to it, some deductive, some inductive, the conclusion having far more content in it than the premises, in that the universal law of gravitation applies to all bodies everywhere, at all times and places, and not just to the bodies of the solar system. It is this inductive, content-increasing character of the argument which introduces the possibility that the conclusion may contradict the initial assumptions, something which is wholly prohibited in a purely deductive argument.

\footnotetext{
${ }^{6}$ See Note 1.
}

A deductive argument is valid if it reveals that it is not possible for the premise to be true and the conclusion false. That is the only requirement. In order to procure validity, the conclusion must be, as it were, contained in the premise. The conclusion must not assert anything in addition to what the premise asserts for, if it did, then it would be possible for that extra bit to be false even though the premise is true, and then the argument would violate the only requirement for validity.

But none of this applies to inductive arguments. The whole point of an inductive argument is to arrive at a universal law or theory from particular observations, experimental results or regularities. Newton exhibits the pattern of an inductive argument very well. In the case of an inductive argument, then, the conclusion asserts far, far more than the premise, and so it is quite possible that the premise might be true and the conclusion false.

Duhem noticed that Newton suffers from a somewhat more serious flaw: the conclusion is actually inconsistent with the premise. One might have thought that it would be reasonable to hold that an inductive argument can only be acceptable as an inductive argument if the premise can be deductively derived from the conclusion - or at least that conclusion and premise are consistent with one another. But, if we take Newton's inductive argument to be acceptable, then we have to acknowledge that these requirements for acceptability are too strong.

We can, however, demand a slightly weaker version of the above requirement. An inductive argument which is such that the conclusion is incompatible with the premise is only acceptable if a modified version of the premise can be derived from the conclusion (and, possibly, other relevant propositions), and this is found to be more accurate by observation or experiment than the original premise. (This is put forward as a necessary, but not a sufficient, requirement for acceptability.)

Newton's inductive argument in the Principia satisfies this weaker requirement for an inductive argument to be acceptable, and it is on that ground that Duhem's criticism is to be rebutted $^{7}$.

But this reason for rejecting Duhem's criticism does seem somewhat formal and pedantic. That Newton's theory corrects the phenomena upon which it is inductively based is treated as a problem that can be overcome when it ought to be treated as an immense success of the theory, that provides especially strong grounds for its acceptance. Almost everyone agrees that the capacity of a new theory to predict new phenomena, or to predict hitherto unsuspected inaccuracies in accepted laws, provides especially strong grounds for accepting the new theory. The above rebuttal of Duhem's criticism does not seem to do justice to this widely accepted feature of scientific method and rationality.

Perhaps this cannot be done within the constraints of an inductive construal of scientific method. It may be that we need to adopt a hypothetico-deductive, or falsificationist, approach to scientific method in order to do justice to the way a new theory receives especially strong grounds for acceptance from correcting empirical laws upon which it is ostensibly based. Let us look at Popper's criticism of Newton, and induction.

\section{Popper's Criticism of Newton}

Karl Popper reiterated Duhem's criticism of Newton's induc-

${ }^{7}$ Harper (2011: pp. 127-130), also rejects Duhem's criticism of Newton, but not on quite the same grounds as suggested here. 
tive argument and, in some respects, extended it. Popper points out that Galileo's law that freely falling bodies fall with constant acceleration is contradicted by Newton's law of gravitation (plus laws of motion). As a body falls, it draws closer to the centre of the earth, the force due to gravity increases slightly, and so the acceleration increases slightly too ${ }^{8}$. This Popperian extension of Duhem's criticism seems a bit beside the point as Newton does not include Galileo's law among his "phenomena". He alludes to it, for example in proposition 6 of Book $3^{9}$, but only in the valid form that bodies fall equal distances in equal times (which does not require constant acceleration).

Popper would also, however, criticize Newton on rather different and much more general grounds (grounds with which Duhem might not have agreed). Popper argued that no inductive argument is acceptable, whether its conclusions are compatible with its premises or not; and so Newton's inductive argument is not acceptable either, in whatever form. It might seem that there is not much difference between induction and hypothetico-deductivism, as long as inductivism makes no claims to establish laws and theories with certainty, or with some degree of probability. Any inductive argument for a theory $\mathrm{T}$ could, it seems, be reformulated as a hypothetico-deductive reason for accepting $\mathrm{T}$, and vice versa. Both involve accepting $\mathrm{T}$ in the light of evidence that can be derived from $\mathrm{T}$ (ignoring the case of evidence contradicting T). Popper would have disagreed. He would argue as follows. In order to assess the acceptability of a law or theory in the light of evidence it is essential to know whether the evidence has been obtained to test severely the law or theory. If the theory has been subjected to a programme of severe attempted falsification, and has survived, it deserves to be tentatively accepted; if it has not, it does not. No inductive argument can capture this vital dimension of severe attempted falsification, and hence no inductive argument can provide acceptable grounds for accepting a law or theory in science $^{10}$.

Instead of construing scientific method as a process of arriving at theories by induction from phenomena we need rather to conceive of it as a process of proposing ever more bold, ever more falsifiable, conjectures which are then subjected to sustained, fierce attempted empirical refutation. Observations and experiments need to be understood in a theoretical context, as a part of the attempt to falsify theories, or decide between competing theories, and not as data gathered in a theoretically neutral context, to be used subsequently as premises for inductive arguments deployed to establish new theories. Newton's Principia would have been a more scientifically rigorous book if it had put forward the three laws of motion and the law of universal gravitation as pure conjectures from which a wealth of empirically successful predictions are derived. That some of these predictions correct hitherto accepted empirical laws poses no problem whatsoever, and is quite straightforwardly a great success for the theory, in that it indicates that what the theory asserts to exist - every particle of matter attracted by every other particle of matter in accordance with the law of gravitation-really does obtain in Nature ${ }^{11}$.

\footnotetext{
${ }^{8}$ Popper (1972: ch. 5)

${ }^{9}$ Newton (1962: Vol. 2, p. 411).

${ }^{10}$ Popper $(1959 ; 1963)$

${ }^{11}$ It is not especially anachronistic to suggest that Newton might have formulated the Principia in this hypothetico-deductive mode. Galileo put forward some of his discoveries in this way-for example his theory of sunspots, and his theory of free fall.
}

Newton did not proceed in this way, it may be argued, for purely personal reasons. He hated controversy. If he had formulated the Principia as a hypothetico-deductive treatise, inevitably he would have invited a torrent of criticism. The entire argument would have been attacked as entirely invalid. It has the form: If P then Q; but Q; therefore P. Newton's contemporaries knew very well that this is an invalid mode of argument. That endlessly many true consequences can be derived from Newton's laws does not mean that those laws are true. The Principia would have also been attacked on the grounds that it is based on an absurdity: a purely speculative law which postulates a force - the force of gravity - that acts at a distance through empty space.

Well aware of the highly controversial character of what he sought to establish, Newton formulated his Principia in such a way that criticism was, as far as possible, forestalled from the outset. The phenomena were undeniable. The mathematical theorems were undeniable. Newton did not put forward conjectures. He derived his law of gravitation from the phenomena by means of a rigorous process of induction.

As it was, the Principia did receive criticism. Thus Huygens in a letter to Leibniz declared "Concerning the Cause of the flux given by M. Newton, I am by no means satisfied [by it], nor by all the other theories he builds upon his Principle of Attraction, which seems to me absurd. I have often wondered how he could have given himself all the trouble of making such a number of investigations and difficult calculations that have no other foundation than this very principle"12. Huygens in fact agreed that Newton had shown that planets move so as to be subject to a force that varies inversely with the square of the distance to the sun. What he rejected was the Newtonian thesis that every particle of matter attracts every other particle in the universe in accordance with Newton's law of gravitation ${ }^{13}$.

Can Newton's inductive argument be rescued from the general Popperian criticism indicated above? A case can certainly be made out that Newton's argument for his universal law of gravitation would have been far less persuasive, in the context in which it was put forward, if it had been couched in a hypothetico-deductive form. The deductive-inductive argument that Newton in fact spelled out in the Principia had an astonishing impact. It led to the view among Newton's contemporaries and successors that something quite unprecedented had been achieved: secure knowledge of the inner workings of nature had almost miraculously been acquired for the first time in the history of humanity. Natural philosophy now had a method to follow which led undeniably to authentic knowledge: Newton's deductive and inductive methodology. Those who followed Newton-Euler, Lagrange, Laplace, and many otherscould be confident that the Principia provided a secure basis for elaboration and development. Newton's deductive-inductive argument created an intellectual atmosphere which made it possible for something like the puzzle solving of Kuhnian normal science to ensue - no doubt required if classical physics was to be developed to reveal its full, extraordinary theoretical and empirical scope and success. The subsequent success of Newtonian science owed much, it could be argued, to the fact that Newton formulated his argument for his universal law of gravitation in its actual deductive-inductive form, as opposed to a hypothetico-deductive form.

According to the Popperian criticism of induction indicated

\footnotetext{
${ }^{12}$ Koyré (1965: pp. 117-118).

${ }^{13}$ See Harper (2011: p. 205).
} 
above, hypothetico-deductivism encourages criticism and attempted falsification, and thus encourages the growth of knowledge, whereas inductivism creates the false impression that what has been established inductively constitutes secure knowledge, and thus does not require criticism, and does not need to be subjected to attempted empirical refutation. In thus inhibiting criticism and attempted falsification, inductivism will tend to inhibit the growth of knowledge.

But the historical record does not seem to provide grounds for holding that any of this actually occurred. Newtonian theory was not protected from empirical refutation as a result of natural philosophers adopting a too reverential attitude towards the theory - not at least until the discovery of the anomalous motion of Mercury in the 19th century. And in any case, in order to subject a theory to attempted empirical falsification, what matters is that empirical consequences are derived from the theory and then put to the test of observation and experiment. It does not matter much what attitude of mind those who do the work have - inductive or hypothetico-deductive - as long as a variety of empirical implications of the theory are unravelled, and then tested observationally or experimentally. Those who came after Newton most certainly did apply the theory to as wide a range of phenomena as possible, thereby subjecting the theory to severe empirical testing even if this was not explicitly the intention.

The advance of classical physics after Newton was it seemed helped by general acceptance of Newtonian inductivism, and not retarded in the way Popper's argument would seem to suggest it ought to have been.

It might nevertheless be argued that there would have been substantial benefits if Newton had put forward his law of gravitation as a conjecture which predicts - and thus potentially explains - a wealth of empirical phenomena. The whole subsequent conception of science would have been different. Instead of science being conceived of as a massive, unassailable authority providing knowledge securely established by evidence- as it tended to be viewed in the 19th and early 20th centuries - science would have been understood to be the enterprise of proposing bold, imaginative conjectures which are then subjected to an onslaught of attempted empirical refutation and severe criticism. Scientific knowledge is just our very best guesses about the nature of things that have so far survived our most ferocious attempts to overthrow them. Science would have been more honest, more openly fallible, and such that it had much to teach other areas of human life: politics, economics, even philosophy.

\section{Third Argument Concerning the Metaphysical Presuppositions of the Methods of Physics}

So far I have argued that Duhem's and Popper's criticisms of Newton's deductive-inductive argument for his law of gravitation in the Principia are not perhaps as devastating as one might at first suppose. There is, however, another criticism of Newton's argument that really is devastating. This comes from a line of argument $I$ have developed in recent decades in a series of publications ${ }^{14}$. Granted that this argument is valid, not only must we reject Newton's claim to have derived his law of gravitation from the phenomena by induction without resource to hypotheses. We must also reject Duhem's and Popper's con-

${ }^{14}$ See Maxwell (1974; 1993; 1998; 2000; 2002; 2004; 2005; 2007, especially, ch. $14 ; 2011 ; 2013)$. ceptions of science as well. Indeed, we must reject all conceptions of scientific method, whether inductivist or hypotheticodeductivist, which fail to acknowledge that metaphysical hypotheses play an essential and permanent role in science in the context of acceptance of laws and theories.

My argument can be formulated as follows. Let $\mathrm{T}$ be any accepted fundamental physical theory-Newtonian theory, classical electrodynamics, quantum theory, general relativity, quantum electrodynamics, quantum electroweak theory, quantum chromodynamics, or the standard model. There are, to begin with, infinitely many disunified rivals to $\mathrm{T}-\mathrm{T}_{1}, \mathrm{~T}_{2}, \ldots \mathrm{T}_{\infty}-$ that are just as empirically successful as T. In order to concoct such a rival, $\mathrm{T}_{1}$ say, all we need to do is modify $\mathrm{T}$ in an entirely ad hoc way for phenomena that occur after some future date. Thus, if $\mathrm{T}$ is Newtonian theory (NT), $\mathrm{NT}_{1}$ might assert: everything occurs as NT predicts until the first moment of 2050 (GMT) when an inverse cube law of gravitation comes into operation: $F=G_{1} m_{2} / d^{3}$. Infinitely may such disunified rivals can be concocted by choosing infinitely many different future times for an abrupt, arbitrary change of law. These theories will no doubt be refuted as each date falls due, but infinitely many will remain unrefuted ${ }^{15}$. We can also concoct endlessly many disunified rivals to $\mathrm{T}$ by modifying the predictions of $\mathrm{T}$ for just one kind of system that we have never observed. Thus, if $\mathrm{T}$ is, as before, NT, then $\mathrm{NT}_{2}$ might assert: everything occurs as NT predicts except for any system of pure gold spheres, each of mass greater than 1000 tons, moving in a vacuum, centres no more than 1000 miles apart, when Newton's law becomes F = $\mathrm{Gm}_{1} \mathrm{~m}_{2} / \mathrm{d}^{4}$. Yet again, we may concoct further endlessly many equally empirically successful disunified rivals to $\mathrm{T}$ by taking any standard experiment that corroborates $\mathrm{T}$ and modifying it in some trivial, irrelevant fashion - painting the apparatus purple, for example, or sprinkling diamond dust in a circle around the apparatus. We then modify $\mathrm{T}$ in an ad hoc way so that the modified theory, $\mathrm{T}_{3}$ say, agrees with $\mathrm{T}$ for all phenomena except for the trivially modified experiment. For this experiment, not yet performed, $\mathrm{T}_{3}$ predicts - whatever we choose. We may choose endlessly many different outcomes, thus creating endlessly many different modifications of $\mathrm{T}$ associated with this one trivially modified experiment. On top of that, we can, of course, trivially modify endlessly many further experiments, each of which generates endlessly many further disunified rivals to T.

Each of these equally empirically successful, disunified rivals to $\mathrm{T}-\mathrm{T}_{1}, \mathrm{~T}_{2}, \ldots \mathrm{T}_{\infty}$-can now be modified further, so that each becomes empirically more successful than T. Any accepted fundamental physical theory is almost bound to face some empirical difficulties, and is thus, on the face of it, refuted -by phenomena A. There will be phenomena, B, which come within the scope of the theory but which cannot be predicted

\footnotetext{
${ }^{15}$ The famous arguments of David Hume (1959) concerning causation have long rendered familiar the point that, for all we can ever know for certain, physical laws may, at some future time, abruptly change, or, to put the same point in an equivalent way, given any physical theory, however empirically successful, there will always be infinitely many rival theories that fit all available evidence just as well but which postulate an abrupt change in physical laws at some future time. What I go on to argue in the text is that these "Humean" theories, as we may call them, that postulate an abrupt change in physical law at some future time are just special cases of a much wider class of disunified theories that postulate an abrupt change in physical law as conditions change in some way other than time, such as kind of system, or experimental conditions. And furthermore, I argue, all these disunified theories can be further doctored to be empirically more successful than the theories we accept.
} 
because the equations of the theory cannot (as yet) be solved. And there will be other phenomena $(\mathrm{C})$ that fall outside the scope of the theory altogether. We can now take any one of the disunified rivals to $\mathrm{T}, \mathrm{T}_{1}$ say, and modify it further so that the new theory, $\mathrm{T}_{1}{ }^{*}$, differs further from $\mathrm{T}$ in predicting, in an entirely ad hoc way, that phenomena $\mathrm{A}, \mathrm{B}$ and $\mathrm{C}$ occur in accordance with empirically established laws $\mathrm{L}_{\mathrm{A}}, \mathrm{L}_{\mathrm{B}}$ and $\mathrm{L}_{\mathrm{C}} \cdot \mathrm{T}_{1}{ }^{*}$ successfully predicts all that $\mathrm{T}$ has successfully predicted; $\mathrm{T}_{1}{ }^{*}$ successfully predicts phenomena $\mathrm{A}$ that ostensibly refute $\mathrm{T}$; and $\mathrm{T}_{1}{ }^{*}$ successfully predicts phenomena $\mathrm{B}$ and $\mathrm{C}$ that $\mathrm{T}$ fails to predict. On empirical grounds alone, $\mathrm{T}_{1}{ }^{*}$ is clearly more successful and better corroborated, than $\mathrm{T}$. And all this can be repeated as far as all the other disunified rivals of $\mathrm{T}$ are concerned, to generate infinitely many empirically more successful disunified rivals to $\mathrm{T}: \mathrm{T}_{1}{ }^{*}, \mathrm{~T}_{2}{ }^{*}, \ldots \mathrm{T}_{\infty}{ }^{*} 16$.

But even though all of $\mathrm{T}_{1}{ }^{*}, \mathrm{~T}_{2}{ }^{*}, \ldots \mathrm{T}_{\infty}{ }^{*}$ are more successful empirically than $\mathrm{T}$, they are all, quite correctly, ignored by physics because they are all horribly disunified ${ }^{17}$. They postulate different laws for different phenomena in a wholly ad hoc fashion, and are just assumed to be false. But this means physics makes a big, implicit assumption about the universe: it is such that all such grossly disunified, "patchwork quilt" theories are false.

If physicists only ever accepted theories that postulate atoms even though empirically more successful rival theories are available that postulate other entities such as fields, it would surely be quite clear: physicists implicitly assume that the universe is such that all theories that postulate entities other than atoms are false. Just the same holds in connection with unified theories. That physicists only ever accept unified theories even though endlessly many empirically more successful, disunified rival theories are available means that physics implicitly assumes that the universe is such that all such disunified theories are false.

In accepting the (more or less) unified theories that it does accept-Newtonian theory, classical electrodynamics and the rest - physics thereby adopts a big, highly problematic metaphysical hypothesis, $\mathrm{H}$, about the nature of the universe: it is such that all rival, grossly disunified, "patchwork quilt" but empirically more successful theories are false. H, though a metaphysical hypothesis, is nevertheless a permanent, even if generally unacknowledged, item of theoretical knowledge. Theories that clash with it, even though empirically more successful than accepted physical theories, are rejected - or rather, are not even considered for acceptance. Whenever a fundamental physical theory is accepted, endlessly many empirically more successful rivals, easily formulated, are not even considered just because, in effect, they clash with $\mathrm{H}$. Thus $\mathrm{H}$ is a permanent item of theoretical knowledge in physics, more securely established in scientific practice indeed than any physical theory. Physical theories tend eventually to be shown to be false, but $\mathrm{H}$ persists through theoretical revolutions in physics ${ }^{18}$.

Nevertheless, $\mathrm{H}$ is a hypothesis, a pure conjecture. How can one make sense of the idea that science is rational and delivers authentic knowledge if the whole enterprise depends crucially

\footnotetext{
$\overline{{ }^{16} \text { Even if no phenomena ostensibly refute } \mathrm{T} \text {, each of } \mathrm{T}_{1}{ }^{*}, \mathrm{~T}_{2}{ }^{*}, \ldots \mathrm{T}_{\infty}{ }^{*} \text { is still }}$ more successful empirically than T.

${ }^{17}$ For my account of theoretical unity, an account which emerges from the argument expounded in the text, see Maxwell (1998, Chs. 3 and 4; 2004, Appendix, Section 2; 2007: pp. 373-386; 2013, Section 4).

${ }^{18}$ For expositions of this argument see Maxwell (1974: Part 1; 1993: Part 1; 1998: ch. $2 ; 2000 ; 2002 ; 2004$, ch. $1 ; 2005 ; 2011 ; 2013$.
}

on accepting such an unsupported hypothesis as a secure item of scientific knowledge - a hypothesis that exercises a major influence over what theories are accepted and rejected in physics?

\section{Aim-Oriented Empiricism}

In order to answer this question, we need to adopt a conception of science that I have called "aim-oriented empiricism". Precisely because $\mathrm{H}$ is a substantial assertion about the nature of the universe, an assertion that, though purely conjectural in character, nevertheless exercises a major influence over what theories are accepted and rejected, even to the extent of overriding empirical considerations, it needs to be made explicit within physics so that it can be critically assessed, rival hypotheses if possible being developed and assessed, in the hope that $\mathrm{H}$ can be improved on. We need a new conception of science, I have argued, which represents the metaphysical hypotheses of physics in the form of a hierarchy of hypotheses, as one goes up the hierarchy hypotheses becoming less and less substantial, and more nearly such that their truth is required for science, or the pursuit of knowledge, to be possible at all. In this way we create a relatively unproblematic framework of hypotheses, and associated methodological rules, high up in the hierarchy, within which much more substantial and problematic hypotheses, and associated methodological rules, low down in the hierarchy, can be critically assessed and, we may hope, improved, in the light of the empirical success they lead to, and other considerations: see Figure 1.

At the top there is the relatively insubstantial hypothesis that the universe is such that we can acquire some knowledge of our local circumstances. If this hypothesis is false, we will not be able to acquire knowledge whatever we assume. We are justified in accepting this hypothesis permanently as a part of our knowledge, even though we have no grounds for holding it to be true. As we descend the hierarchy, the hypotheses become

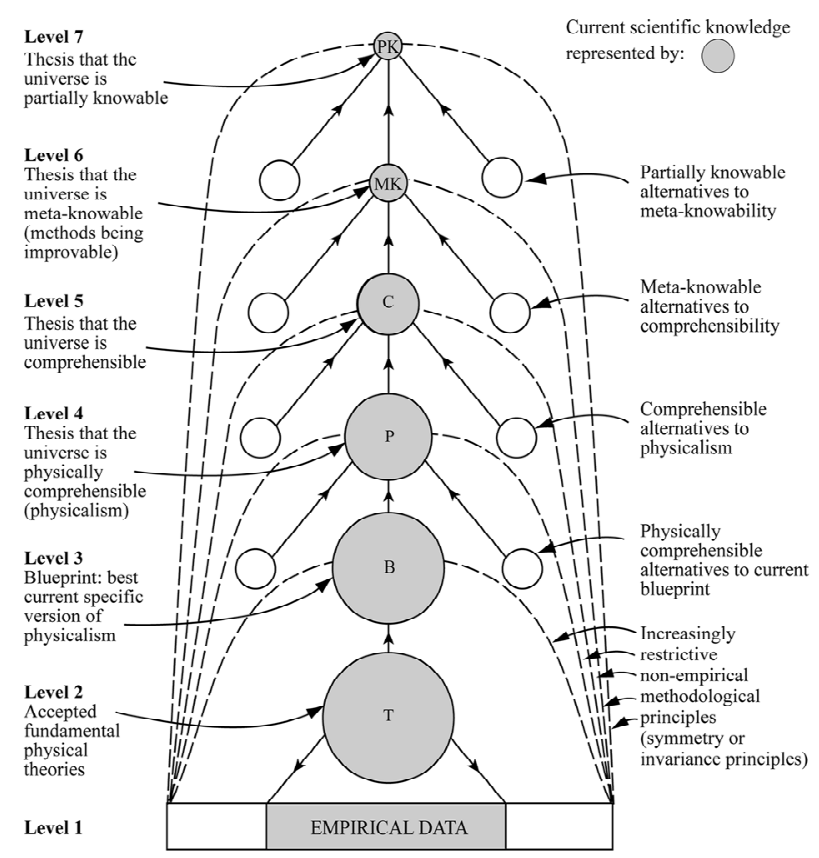

Figure 1.

Aim-Oriented Empiricism (AOE). 
increasingly substantial and thus increasingly likely to be false. At level 6 there is the more substantial hypothesis that the universe is such that there is some rationally discoverable thesis about the nature of the universe which, if true and if accepted, makes it possible progressively to improve methods for the improvement of knowledge. "Rationally discoverable", here, means at least that the thesis is not an arbitrary choice from infinitely many analogous theses. At level 5 we have the even more substantial hypothesis that the universe is comprehensible in some way or other, whether physically or in some other way. This hypothesis asserts that the universe is such that there is something (God, tribe of gods, cosmic goal, physical entity, cosmic programme or whatever), which exists everywhere in an unchanging form and which, in some sense, determines or is responsible for everything that changes (all change and diversity in the world in principle being explicable and understandable in terms of the underlying unchanging something). A universe of this type deserves to be called "comprehensible" because it is such that everything that occurs, all change and diversity, can in principle be explained and understood as being the outcome of the operations of the one underlying something, present throughout all phenomena. At level 4 we have the still more substantial hypothesis that the universe is physically comprehensible in some way or other. This asserts that the universe is made up one unified self-interacting physical entity (or one kind of entity), all change and diversity being in principle explicable in terms of this entity. What this amounts to is that the universe is such that some yet-to-be-discovered unified physical theory of everything is true. At Level 3, we have an even more substantial hypothesis, the best, currently available specific idea as to how the universe is physically comprehensible. This asserts that everything is made of some specific kind of physical entity: corpuscle, point-particle, classical field, quantum field, convoluted space-time, quantum string, or whatever. Given the historical record of dramatically changing ideas at this level, and given the relatively highly specific and substantial character of successive hypotheses made at this level, we can be reasonably confident that the best hypothesis available at any stage in the development of physics at this level will be false, and will need future revision. Here, ideas evolve with evolving knowledge. At Level 2 there are the accepted fundamental theories of physics, currently general relativity and the standard model. Here, if anything, we can be even more confident that current theories are false, despite their immense empirical success. This confidence comes partly from the vast empirical content of these theories, and partly from the historical record. The greater the content of a proposition the more likely it is to be false; the fundamental theories of physics, general relativity and the standard model have such vast empirical content that this in itself almost guarantees falsity. And the historical record backs this up; Kepler's laws of planetary motion, and Galileo's laws of terrestrial motion are corrected by Newtonian theory, which is in turn corrected by special and general relativity; classical physics is corrected by quantum theory, in turn corrected by relativistic quantum theory, quantum field theory and the standard model. Each new theory in physics reveals that predecessors are false. Indeed, if the Level 4 hypothesis is correct, then all current physical theories are false, since this hypothesis asserts that the true physical theory of everything is unified, and the totality of current fundamental physical theory, general relativity plus the standard model, is notoriously disunified. Finally, at Level 1 there are accepted empirical data, low level, corroborated, empirical laws ${ }^{19}$.

The idea is to separate out what is most likely to be true, and not in need of revision, at and near the top of the hierarchy, from what is most likely to be false, and most in need of criticism and revision, near the bottom of the hierarchy. Evidence, at Level 1, and hypotheses high up in the hierarchy, are rather firmly accepted as a part of theoretical knowledge in physics, as being most likely to be true (although still open to revision): this is then used to criticize, and to try to improve, hypotheses at Levels 2 and 3 (and perhaps 4), where falsity is most likely to be located.

In order to be acceptable, an hypothesis at any level from 6 to 3 must (as far as possible) be compatible with, and a special case of, the hypothesis above in the hierarchy; at the same time it must be (or promise to be) empirically fruitful in the sense that successive accepted physical theories increasingly successfully accord with (or exemplify) the hypothesis. At Level 2, those physical theories are accepted which are sufficiently (a) empirically successful and (b) in accord with the best available hypothesis at Level 3 (or Level 4). Corresponding to each hypothesis, at any level from 7 to 3 , there is a methodological principle, represented by sloping dotted lines in the diagram, requiring that hypotheses lower down in the hierarchy are compatible with the given hypothesis.

When theoretical physics has completed its central task, and the true theory of everything, $\mathrm{T}$, has been discovered, then $\mathrm{T}$ will (in principle) successfully predict all empirical phenomena at Level 1, and will entail the hypothesis at Level 3, which will in turn entail the hypothesis at Level 4, and so on up the hierarchy. As it is, physics has not completed its task, T has not (yet) been discovered, and we are ignorant of the nature of the universe. This ignorance is reflected in clashes between theses at different levels of aim-oriented empiricism (AOE). There are clashes between Levels 1 and 2, 2 and 3, and 3 and 4. And the two fundamental theories at Level 2, the standard model and general relativity, clash as well. The attempt to resolve these clashes drives physics forward.

In seeking to resolve clashes between levels, influences can go in both directions. Thus, given a clash between Levels 1 and 2 , this may lead to the modification, or replacement of the relevant theory at Level 2; but, on the other hand, it may lead to the discovery that the relevant experimental result is not correct for any of a number of possible reasons, and needs to be modified. In general, however, such a clash leads to the rejection of the level 2 theory rather than the level 1 experimental result; the latter are held onto more firmly than the former, in part because experimental results have vastly less empirical content than theories, in part because of our confidence in the results of observation and direct experimental manipulation (especially after repetition and expert critical examination). Again, given a clash between Levels 2 and 3, this may lead to the rejection of the

\footnotetext{
${ }^{19}$ This hierarchical conception of scientific method seems first to have been expounded in Maxwell (1974). It was further elaborated in Maxwell (1993). A more elaborate version still is expounded and defended in great detail in Maxwell (1998). For a more detailed defence of the version indicated here, see Maxwell (2004: chs. 1 and 2, and appendix). Maxwell (2005) argues that this view is a sort of synthesis of the views of Popper, Kuhn and Lakatos, but an improvement over the views of all three. Maxwell (2007: ch. 14) gives a detailed exposition of aim-oriented empiricism, and argues, in some detail, that the view succeeds in solving major problems in the philosophy of science, including the problems of induction, simplicity and verisimilitude. The most recent, and possibly the best, exposition and defense of aim-oriented empiricism is Maxwell (2013).
} 
relevant Level 2 theory (because it is disunified, ad hoc, at odds with the current metaphysics of physics); but, on the other hand, it may lead to the rejection of the Level 3 hypothesis and the adoption, instead, of a new hypothesis (as has happened a number of times in the history of physics). The rejection of the current Level 3 hypothesis is likely to take place if the Level 2 theory, which clashes with it, is highly successful empirically, and furthermore has the effect of increasing unity in the totality of fundamental physical theory overall, so that clashes between Levels 2 and 4 are decreased. In general, however, clashes between Levels 2 and 3 are resolved by the rejection or modification of theories at Level 2 rather than the assumption at Level 3, in part because of the vastly greater empirical content of Level 2 theories, in part because of the empirical fruitfulness of the Level 3 assumption (in the sense indicated above).

It is conceivable that the clash between Level 2 theories and the Level 4 hypothesis might lead to the revision of the latter rather than the former. This happened when Galileo rejected the then current level 4 hypothesis of Aristotelianism, and replaced it with the idea that "the book of nature is written in the language of mathematics" (an early precursor of our current Level 4 hypothesis). The whole idea, however, is that as we go up the hierarchy of hypotheses we are increasingly unlikely to encounter error, and the need for revision. The higher up we go, the more firmly hypotheses are upheld, the more resistance there is to modification.

The idea of representing the metaphysical presuppositions of physics (concerning the nature of the universe) as a hierarchy of theses, increasingly insubstantial as one goes up the hierarchy, gains some support from the fact that something somewhat similar exists informally at the empirical level-Level 1 of the figure - and for much the same reason. There are, at the lowest level, the results of experiments performed at specific times and places. Then, above these, there are low-level experimental laws, asserting that each experimental result is a repeatable effect. Next up, there are empirical laws such as Hooke's law, Ohm's law or the gas laws. Above these there are such physical laws as those of electrostatics or of thermodynamics. And above these there are theories which have been refuted, but which can be "derived", when appropriate limits are taken, from accepted fundamental theory-as Newtonian theory can be "derived" from general relativity. This informal hierarchy at the empirical level exists for the same reason we need the hierarchy at the metaphysical level: so that relatively contentless and secure theses (at the bottom of the empirical hierarchy) may be distinguished from more contentful and insecure theses (further up the hierarchy) to facilitate pinpointing what needs to be revised, and how, should the need for revision arise. That such a hierarchy exists at the empirical level provides some support for my claim that we need to adopt such a hierarchy at the metaphysical level.

AOE, as depicted in Figure 1, provides physics with a meta-methodology which facilitates improvement of the metaphysical hypotheses, and associated methods, as physics advances, in the light of which seem to be the most fruitful empirically, and other considerations. As knowledge in physics improves, so metaphysical hypotheses and methods improve as well or, in other words, we improve knowledge about how to improve knowledge. There is something like positive feedback between improving knowledge of the universe, and improving aims and methods, improving knowledge about how to improve knowledge. Everyone would acknowledge that this kind of positive feedback goes on at the empirical level. New empirical knowledge can lead to new methods, via the development of new instruments, new experimental techniques, which in turn lead to further acquisition of new knowledge. AOE provides methods which facilitate such positive feedback at the metaphysical and theoretical level as well. As we increase our scientific knowledge and understanding of the universe, we increase our knowledge of how to increase knowledge - the very nub of scientific rationality which helps explain the explosive, and apparently ever accelerating, growth of scientific knowledge. We adapt the nature of science to what we find out about the nature of the universe. All this has gone on in science to some extent implicitly: what the adoption of AOE does is to make the implicit explicit-science becoming even more successful as a result.

I provide no grounds whatsoever for holding that the hypotheses at Levels 3 to 7 are true, probably true, or likely to be true. What I do rather is to argue that these hypotheses are the best that are available, granted the current state of knowledge in physics, granted that some metaphysical hypothesis such as $\mathrm{H}$ is implicit in the way physics persistently accepts (more or less) unified theories even though endlessly many empirically more successful disunified rivals are always available, and granted that the aim of physics is to discover the truth about the nature of the universe ${ }^{20}$. Hypotheses at Levels 7 and 6 are permanent items of scientific knowledge. Arguments in support of accepting these hypotheses make no appeal to the empirical success of science. As one goes down the hierarchy, from Levels 5 to 3, it becomes increasingly likely that the hypothesis at that level may need to be revised.

\section{My Criticism of Newton, and of Duhem and Popper}

One upshot of the above argument is the conclusion: no conception of science can be tenable which denies, or fails to acknowledge, that metaphysical hypotheses are a secure, permanent part of scientific knowledge-hypotheses that are implicit in those methods of physics which require sufficiently empirically successful and (more or less) unified theories to be accepted in preference to endlessly many empirically more successful disunified rivals.

Both Duhem and Popper defend conceptions of science that do deny that any such metaphysical hypotheses are an integral part of scientific knowledge. These conceptions of science must thus be rejected.

Newton claims in the Principia to have derived his law of gravitation by induction from the phenomena without adopting any hypotheses. He makes this clear in the General Scholium at the end of the Principia. He says "whatever is not deduced from the phenomena is to be called a hypothesis; and hypotheses, whether metaphysical or physical, whether of occult qualities or mechanical, have no place in experimental philosophy. In this philosophy, particular propositions are inferred from the phenomena, and afterwards rendered general by induction. Thus it was that... the laws of motion and of gravitation were discovered." 21

But Newton is wrong. In establishing his law of gravitation by induction Newton cannot avoid adopting metaphysical hy-

\footnotetext{
${ }^{20}$ See Maxwell (1998: ch. 5; 2004: Appendix, Section 6; 2007: pp. 400-430; 2013: Sections 7 and 8)

${ }^{21}$ Newton (1962: Vol. 2, p. 547).
} 
potheses. These are implicit in his four rules of reasoning in philosophy, quoted above. Infinitely many rivals to Newton's law of gravitation are compatible with all the phenomena Newton cites in the Principia, as the argument of Section 4 above makes clear. Some of these rivals would be even more empirically successful than Newtonian theory-for example, rivals which modify Newtonian theory in a wholly ad hoc way so as to predict the rotation of the perihelion of Mercury's orbit, but otherwise are precisely the same as Newtonian theory. These infinitely many rivals are ruled out of court, not by evidence, not by the phenomena, but by one or other, or all, of Newton's four rules of reasoning - by Newton's conception of scientific method, in other words.

One rival to Newton's law of gravitation might postulate additional forces which cancel themselves out for the solar system but which do not for stars and other heavenly bodies far away, which gravitate towards one another in accordance with a variety of laws. Such a theory (and endlessly many could be concocted along these lines) successfully predicts all the phenomena known to Newton that his theory predicts, but this theory is to be excluded on the grounds that "we are to admit no more causes of natural things than such as are ... sufficient to explain their appearances" (Rule 1).

Another rival theory might agree with Newtonian theory as far as the motions of the planets round the sun are concerned, but postulate somewhat different laws of motion and law of gravitation to predict and explain the motions of the moons of Jupiter and Saturn. Such a theory is however to be excluded because it violates the requirement that, "to the same natural effects we must, as far as possible, assign the same causes" (Rule 2).

And yet another rival theory might postulate that planets other than the earth possess, not Newtonian inertial mass but rather what might be called Galilean inertial mass so that, free of any force, their inertial motion is to go round the sun in accordance with Kepler's laws (or even orbits predicted by Newtonian theory), there thus being no gravitational force between these planets and the sun. Such a theory is however to be ignored because, although it is compatible with the phenomena, it violates the requirement that "the qualities of bodies ... which are found to belong to all bodies within the reach of our experiments, are to be esteemed the universal qualities of all bodies whatsoever" (Rule 3).

Newton's inductive argument of the Principia and his rules of reasoning presuppose that terrestrial and astronomical phenomena obey the same laws, so that one can make inductive inferences from terrestrial to astronomical phenomena. But, given the experimental knowledge available at the time of Newton, this amounts to a substantial metaphysical hypothesis about the nature of the universe. It is a hypothesis denied, for example, by Aristotle. Generalizing inductively from experimental knowledge of terrestrial phenomena to astronomical phenomena everywhere, at all times, presupposes implicitly the truth of this metaphysical hypothesis. Such inductive generalizing will only meet with success if the hypothesis is true. In an Aristotelian universe, it would lead one badly astray.

Newton's claim to have established his law of gravitation from the phenomena by induction without metaphysical hypotheses is thus untenable. Metaphysical hypotheses are implicit in his four rules of reasoning, and these rules play a crucial role in his inductive argument for his law of gravitation.
But, as we saw in Section 4 above, this holds for all methods of physics which exclude from consideration theories that are disunified, ad hoc or complex, whatever their potential empirical success might be. 20th and 21 st century views about scientific method are just as vulnerable to the criticism as Newton's view is.

In some respects, indeed, Newton does better than contemporary scientists and philosophers of science. Most 20th and 21 st scientists and philosophers of science acknowledge that considerations of simplicity and unity play a role in physics in deciding what theories are to be accepted and rejected, but nevertheless would deny that this means physics adopts a substantial, permanent metaphysical hypothesis about the nature of the universe as a part of scientific knowledge. Newton is more enlightened. He quite explicitly acknowledges that his rules of reason do make assumptions about the nature of the universe. He acknowledges this in his comments on the first three of his four rules.

Thus, in connection with Rule 1, Newton declares "philosophers say that Nature does nothing in vain, and more is in vain when less will serve; for Nature is pleased with simplicity, and affects not the pomp of superfluous causes",22. In other words, if the universe were such that it delighted in complexity, and constantly affected the pomp of superfluous causes, it would be foolish and counterproductive to adopt Newton's first rule of inductive reasoning. In adopting the rule, and in accepting and rejecting theories in accordance with it, we thereby adopt a substantial hypothesis about the nature of the universe-and Newton, unlike today's scientists, was well aware of this fact.

Again, in connection with Rule 2, Newton comments "As to respiration in a man and in a beast; the light of our culinary fire and of the sun; the reflection of light in the earth, and in the planets" 23 . But only in a certain kind of universe will the same natural effects tend to have the same causes. It is not hard to imagine possible universes where nothing of the kind holds, and yet it is still possible for us to live, and to acquire knowledge $^{24}$. Newton is again more enlightened than most modern scientists and philosophers of science in appreciating that his second rule of inductive reasoning makes an assumption about the nature of the universe which, if false, would render the rule unproductive, the wrong rule to adopt.

And a similar point holds in connection with Newton's much lengthier comments on his third rule ${ }^{25}$. Here, too, Newton makes clear that this rule makes a substantial assumption about the nature of the universe, and will only be a sensible rule to adopt in so far as the universe accords with what this assumption says about it.

About the forth rule Newton says merely: "This rule we must follow, that the argument of induction may not be evaded by hypotheses" ${ }^{26}$. This is a great disappointment after Newton's enlightened comments on the first three rules. Far from stressing that in adopting and implementing Rule 4 we adopt a metaphysical hypothesis about the nature of the universe, Newton asserts almost the opposite: we must implement Rule 4 to ensure that we do not assume metaphysical hypotheses.

\footnotetext{
${ }^{22}$ Newton (1962: Vol. 2, p. 398).

${ }^{23}$ Newton (1962: Vol. 2, p. 398).

${ }^{24}$ See Maxwell (1998: ch. 5) for imaginative suggestions for possible universes in which science as we know it would not be possible because the metaphysical hypotheses implicit in scientific method are false, but in which life is possible, and the pursuit of knowledge can meet with success.

${ }^{25}$ See Newton (1962: Vol. 2, pp. 398-400).

${ }^{26}$ Newton (1962: Vol. 2, p. 400).
} 
It is significant, however, that Rule 4 only appeared in the third edition of the Principia published in $1726^{27}$. It was, it seems, an afterthought. Years after the publication of the first edition, Newton may have managed to convince himself that no metaphysical hypotheses were involved in his inductive argument for his law of gravitation; or possibly he may have become increasingly concerned to create the impression that that was the case.

It is I suppose possible to interpret Newton's comments on the first three of his four rules of reasoning as mere rhetorical flourishes, signifying nothing very much. Doubtless that is how they came to be interpreted in subsequent decades and centuries. My own view is that we should give Newton the credit of meaning what he said, and he did, in the first edition of the Principia at least, clearly state that his first three rules of reasoning make metaphysical assumptions about the nature of the universe. This means, of course, that what Newton says here is inconsistent with what he claims in the General Scholium (an addition to a later edition) that no hypotheses, metaphysical or physical, were invoked in his inductive derivation of the law of gravitation from the phenomena.

\section{Conclusion}

There are two conclusions.

First, Newton was wrong when he claimed to have derived his law of gravitation from the phenomena by induction without metaphysical hypotheses. On the contrary, in employing his rules of reasoning during the course of his inductive derivation, he thereby did adopt metaphysical hypotheses about the nature of the universe, as he himself in effect acknowledges in his comments the first three of these four rules.

Second, my long-standing argument is correct. No conception of science, and of scientific method, is tenable which denies, or fails to acknowledge, that persistent preference for (more or less) unified theories in physics even when endlessly many empirically more successful disunified rivals are available means that physics adopts a permanent metaphysical hypothesis about the nature of the universe: it is such that all seriously disunified theories are false. Once this point is acknowledged it becomes clear that we need to adopt the aim-oriented empiricist conception of science which displays metaphysical hypotheses of physics in the form of a hierarchy, and thereby maximizes our capacity to develop and select those hypotheses best able to promote scientific progress.

\section{REFERENCES}

Duhem, P. (1962). The aim and structure of physical theory. New York: Atheneum.

Harper, W. L. (2011). Isaac Newton's scientific method. Oxford: Oxford University Press.

Hume, D. (1959). A treatise of human nature. Book 1. London: Everyman.

Koyré, A. (1965). Newtonian studies. London: Chapman and Hall.

Maxwell, N. (1974). The rationality of scientific discovery. Philosophy of Science, 41, 123-153, 247-295. http://dx.doi.org/10.1086/288579

Maxwell, N. (1993). Induction and scientific realism. British Journal for the Philosophy of Science, 44, 61-79, 81-101, 275-305. http://dx.doi.org/10.1093/bjps/44.1.61

Maxwell, N. (1998). The comprehensibility of the universe. Oxford: Oxford University Press.

Maxwell, N. (2000). A new conception of science. Physics World, 13, 17-18.

Maxwell, N. (2002). The need for a revolution in the philosophy of science. Journal for General Philosophy of Science, 33, 381-408. http://dx.doi.org/10.1023/A:1022480009733

Maxwell, N. (2004). Is science neurotic? London: Imperial College Press.

Maxwell, N. (2005). Popper, Kuhn, Lakatos and aim-oriented empiricism. Philosophia, 32, 181-239.

Maxwell, N. (2007). From knowledge to wisdom. London: Pentire Press.

Maxwell, N. (2011). A priori conjectural knowledge in physics. In M. Shaffer, \& M. Veber (Eds.), What place for the a priori (pp. 211-240)? Chicago: Open Court.

Maxwell, N. (2013). Has science established that the universe is physiccally comprehensible? In A. Travena, \& B. Soen (Eds.), Recent advances in cosmology. New York: Nova Publishers Inc.

Newton, I. (1962). Principia, A. Motte's translation revised by F. Cajori. Berkeley: University of California Press.

Popper, K. R. (1959). The logic of scientific discovery. London: Hutchinson.

Popper, K. R. (1963). Conjectures and refutations. London: Routledge and Kegan Paul.

Popper, K. R. (1972). Objective knowledge. London: Oxford University Press.

\footnotetext{
${ }^{27}$ I am grateful to Harper's magnificent and detailed book on Newton's scientific method for this point: see Harper (2011: p. 362).
} 